\title{
ACCIONES Y PROYECTOS DEL GABINETE PEDAGÓGICO DE LA FACULTAD DE BIOQUÍMICA Y CIENCIAS BIOLÓGICAS
}

Manuale, Marcela

El Gabinete Pedagógico desarrolla sus tareas en función de varios ejes: por un lado, el asesoramiento y la capacitación docente, a través de diversos cursos y talleres con diversos destinatarios; por otro lado, se vincula a proyectos de investigación educativa, y también cumple una labor de extensión y comunicación, a través de la revista Aula Universitaria.

La función de asesoramiento específica del asesor pedagógico del Gabinete Pedagógico se desarrolla respecto de cuatro áreas de trabajo en relación con los destinatarios:

* con la institución educativa como organización académica;

* con los docentes;

* con los alumnos;

* con el equipo del Gabinete Pedagógico.

Nos detendremos en las acciones con los alumnos y con los docentes.

Con relación a los alumnos, hay tres líneas principales de trabajo:

- Recepción a los ingresantes en el inicio de cada ciclo lectivo (Jornadas de ambientación y orientación pedagógico-institucional. ingresantes (Res. CD n 577/00), cuyo diseño y desarrollo se comparte con la psicopedagoga Nidia Baumann, las autoridades académicas, los coordinadores de carreras y los docentes de primer año, así como los representantes de los Centros de Estudiantes.

- Suministro de las Fichas psicopedagógicas, que permiten elaborar un perfil de los alumnos ingresantes, desde el punto de vista personal, educativo, vocacional y cultural.

- Elaboración de los Informes de las Fichas Psicodegógicas, que se elevan al Consejo Directivo de la Facultad y a Secretaría Académica, para consulta de los docentes y de la comunidad educativa en general. 
- Propuesta y desarrollo de un Taller de Estrategias de aprendizaje "Aprender a aprender" (Res. CD n 134 - Expte. n 58.665-0/96), desarrollado desde 1996 y que continúa hasta la actualidad todos los años, dirigido a los alumnos de primer año -y que también incluye a alumnos de años superiores-, destinado a construir las herramientas básicas para el estudio universitario. Sus objetivos apuntan a:

- Favorecer la reflexión acerca del sentido, posibilidades y exigencias del trabajo intelectual.

- Introducir a los estudiantes en el dinamismo del trabajo intelectual a través de la práctica -individual y grupal- de diferentes operaciones/procedimientos, y de la reconstrucción y evaluación de los mismos.

- Posibilitar la comprensión y sistematización de algunas categorías de análisis que permiten la interacción con los saberes vigentes.

Además planteamos algunos objetivos específicos que puedan concretar la construcción de un aprendizaje estratégico:

- Desarrollar un proceso de conocimiento y control de estrategias de aprendizaje cognitivas (de elaboración, de organización y de recuperación), de estrategias metacognitivas (para el conocimiento de la persona, la tarea y las estrategias utilizadas, y el control de la planificación, la supervisión y la evaluación del propio aprendizaje); y de estrategias motivacionales (focalizar la atención, diseñar actividades y favorecer la motivación intrínseca).

- Promover estrategias cognitivas y metacognitivas para la comprensión lectora.

En el año 2009 los Talleres también incorporaron a los tutores del Programa PACENI.

\section{En relación con los docentes}

Con respecto a la capacitación docente, las acciones del Gabinete Pedagógico se dirigen a los docentes universitarios que tienen serias falencias en su formación pedagógico-didáctica.

Como se afirma en la propuesta del Gabinete Pedagógico, el perfeccionamiento docente es una cuestión crucial, ya que "los docentes universitarios son uno de los principales destinatarios de las acciones del Gabinete Pedagógico, lo que plantea un trabajo complejo debido a las siguientes razones:

* los profesores universitarios están ubicados en el nivel más alto del sistema educativo, lo que implica un nivel de formación exigente;

* la formación profesional específica de los bioquímicos y de las ciencias biológicas no los ha capacitado pedagógicamente; 
* el aislamiento que impone el trabajo en el aula, que muchas veces dificulta la formulación de nuevos proyectos e innovaciones;

* el deterioro de la función docente que imponen las actuales políticas educativas y económicas".

Así, se proponen las siguientes estrategias de intervención.

"Líneas de acción:

A) Con relación a los docentes (cátedras y departamentos)

La direccionalidad del trabajo con este sector está dada por la formación, capacitación y perfeccionamiento de los docentes universitarios en tanto educadores del nivel superior. La formación pedagógica es un objetivo prioritario.

Entendemos la enseñanza como una actividad social que requiere respuestas diferenciadas, tanto en los contenidos que se enseñan como en sus estrategias didácticas.

Por lo tanto, la capacitación no puede plantearse fuera del contexto, sino inserta en un puesto de trabajo socialmente determinado.

Entendemos que la capacitación docente debe permitir la reflexión crítica de las prácticas vigentes, recuperando al docente como un profesional creativo, constructor del conocimiento, superando la vieja concepción de un profesional como mero transmisor de contenidos ya elaborados. Por lo tanto, la asesoría pedagógica debe generar espacios donde los docentes puedan revisar y comprender sus prácticas, para poder modificarlas.

De allí que la investigación y evaluación de las prácticas académicas (la organización curricular, metodología del proceso de enseñanza-aprendizaje, sistemas de práctica, formas de evaluación...) que se deberá promover, implicarán un conjunto de acciones que aporten a un trabajo intelectual autónomo y creativo.

Así, se prevé la organización de diferentes instancias:

- Reuniones de los docentes con el gabinete, por curso y por áreas, para intercambiar demandas y problemáticas con respecto al proceso de enseñanza y de aprendizaje.

- Organización de talleres de reflexión de las prácticas docentes, para analizar críticamente los supuestos subyacentes de su tarea.

- Organización de paneles, cursos, charlas, talleres, etc. -con recursos humanos de la casa o con especialistas invitados- sobre temáticas de interés acordadas con los docentes.

- Desarrollar acciones tendientes a la apropiación de estrategias didácticas, no sólo como elaboración de técnicas e instrumentos, sino en un nivel más complejo 
de reconstrucción teórica del objeto de conocimiento con la intencionalidad de ser enseñado.

- Elaborar conjuntamente pautas para la planificación didáctica y la evaluación de procesos y resultados.

La modalidad de las acciones cumple diferentes objetivos: tomar conciencia de las problemáticas, generar espacios y formas de trabajo sobre ellas y acceder a construcciones teóricas y pedagógicas-didácticas que puedan fundamentar los mejoramientos que se planteen.

Sintetizando, el propósito del asesoramiento y supervisión pedagógica será promover en los docentes hábitos de reflexión con respecto a la práctica de la enseñanza, para estimular un mejor aprendizaje en los alumnos."

Estas líneas se han desarrollado en función de las demandas de diversas cátedras que requieren del asesoramiento pedagógico o con docentes particulares que lo solicitan en forma personal, ya sea como una formación individual o para desarrollar proyectos de sus cátedras de origen.

Las tareas de asesoramiento tienen que ver fundamentalmente con las planificaciones didácticas, consultas sobre metodologías de enseñanza o de evaluación. También las consultas se han referido al diseño de los programas de cátedra, en ocasión de los numerosos concursos docentes desarrollados en nuestra unidad académica.

Además del asesoramiento a las cátedras, se han propuesto diversos cursos o talleres de reflexión de diversas problemáticas pedagógico-didácticas. Estos cursos se han desarrollado mayoritariamente como cursos de extensión, en forma presencial, en la misma unidad académica. Los cursos o talleres se plantearon sin cargo para los docentes de la casa y con un costo mínimo para docentes de otras unidades académicas.

\section{Propuestas de capacitación docente}

Durante 2008 y 2009, y como responsable del Gabinete Pedagógico, he desarrollado diversas propuestas de extensión y capacitación docente y de docencia de posgrado:

- Dictado del curso de capacitación docente: "Estrategias de aprendizaje para favorecer los procesos comprensivos", destinado a docentes de todos los niveles, en el marco del Cursos de Extensión a distancia de la UNL, desde mayo a julio de 2008, con un total de 110 horas, con evaluación final (aprobado por los ministerios de educación de las provincias de Santa Fe, Entre Ríos y Córdoba y en trámite en Buenos Aires y San Luis) (RM nº 942 del 18 Julio de 2008, se reconoció como Ac- 
tividad de la Cabecera Provincial de la Red Federal de Formación Docente Continua, por la Dirección Provincial de Innovación, Actualización y Evaluación Educativa).

- Dictado del curso de capacitación docente: "Enseñanza para la Comprensión: ¿cómo mejorar el aprendizaje y la evaluación de nuestros alumnos?", destinado a docentes de todos los niveles, en el marco del Cursos de Extensión a distancia de la UNL, desde julio a septiembre de 2008, con un total de 110 horas, con evaluación final (aprobado por los ministerios de educación de las provincias de Santa Fe, Entre Ríos y Córdoba y en trámite en Buenos Aires y San Luis) (RM n ${ }^{\circ}$ 942 del 18 Julio de 2008, se reconoció como Actividad de la Cabecera Provincial de la Red Federal de Formación Docente Continua, por la Dirección Provincial de Innovación, Actualización y Evaluación Educativa).

- Dictado del curso de capacitación docente a distancia: "Planificación didáctica: una tarea intelectual del docente, destinado a docentes de todos los niveles", organizado por la Secretaría de Extensión de la UNL, desarrollado en el marco del Programa de Educación a distancia de la UNI (UNL Virtual), aprobado por Resolución del HCS n 464/08, y dictado del 4 de mayo al 7 de agosto de 2009.

- Dictado del curso de capacitación docente a distancia: "Estrategias didácticas: una construcción del docente", destinado a docentes de todos los niveles, organizado por la Secretaría de Extensión de la UNL, desarrollado en el marco del Programa de Educación a distancia de la UNI (UNL Virtual), aprobado por Resolución del HCS n 464/08, y dictado desde julio a diciembre de 2009, con un total de 110 horas, con evaluación final.

- Dirección del Curso de "Estrategias de Trabajo en el aula" de la Maestría en Didáctica de las Ciencias Experimentales de la Facultad de Bioquímica y Ciencias Biológicas de la UNL, desde 2001 y continúa.

- Dictado del curso de Posgrado de Perfeccionamiento "Nuevas tecnologías aplicadas a la enseñanza 2" (aprobado por Res. CD n 335/09) en la Facultad de Bioquímica y Ciencias Biológicas, desde el 13 de mayo hasta el 1 de julio de 2009, en el marco del CAI+D 2009 "Incorporación de nuevas tecnologías para comprensión y análisis crítico de modelos en ciencias", con una carga horaria de 20 horas.

- Dirección del Curso de "Estrategias de Trabajo en el aula" en el marco del Programa PACENI-UNNE (RES. no 1031/09), en la Facultad de Ciencias Exactas y Naturales y Agrimensura de la Universidad Nacional del Noroeste, Corrientes, 15, 16 y 17 de octubre de 2009, con una duración de 50 horas. 


\section{Publicación del Gabinete Pedagógico: Aula Universitaria}

Desde 1996 y en forma continua, la revista Aula Universitaria se ha venido desarrollando como una propuesta de comunicación de las experiencias didácticas innovadoras y las investigaciones pedagógicas generadas desde las diversas cátedras de nuestra Facultad como de otras unidades académicas, ya que se ha expandido el radio de influencia de las producciones. El crecimiento del número y calidad de los trabajos presentados da muestra de la significación de esta revista en el actual contexto universitario.

\section{Trabajo de investigación educativa}

Como parte de la tarea de investigación, el Gabinete Pedagógico participa en el apoyo a proyectos de investigación educativa (CAI+D 2009 "Incorporación de nuevas tecnologías para comprensión y análisis crítico de modelos en ciencias") así como en la dirección de tesis.

Por otra parte, se está diseñando un proyecto de investigación sobre "Las prácticas de enseñanza en la universidad", que está destinado a indagar los estilos y significaciones de la docencia universitaria. 